SHORT REPORT

\title{
Clinical, molecular, and PET study of a case of aceruloplasminaemia presenting with focal cranial dyskinesia
}

\author{
I Haemers, S Kono, S Goldman, J D Gitlin, M Pandolfo
}

J Neurol Neurosurg Psychiatry 2004;75:334-337. doi: 10.1136/jnnp.2003.017434

\begin{abstract}
Aceruloplasminaemia is a rare recessive disorder caused by mutations in the gene encoding the multicopper ferroxidase ceruloplasmin, thought to be involved in cellular iron export. Primary intracellular iron accumulation characterises this disorder. We investigated a case of aceruloplasminaemia early in the course of the disease by structural and functional neuroimaging and correlated the results with the clinical findings. The patient, a diabetic 59 year old lady, presented with perioral dyskinesia. Magnetic resonance imaging (MRI) revealed massive iron accumulation in the basal ganglia, notably sparing the pallidum, and along the cortical surface. However, most of these structures had preserved metabolic activity as evaluated by fluorodeoxyglucose positron emission tomography (FDG-PET). Voxel based analysis of FDGPET data showed a significant hypometabolism only in the heads of the caudate nuclei. Molecular genetic analysis revealed compound heterozygosity for two null mutations in the ceruloplasmin gene, a rather surprising finding for a very rare recessive disease, suggesting that aceruloplasminaemia could be somewhat more frequent than is commonly thought and could therefore be underdiagnosed.
\end{abstract}

the cell surface by a glycosylphosphatidylinositol (GPI) anchor. ${ }^{8}$ This GPI anchored form of ceruloplasmin is likely to be the major form of this molecule in the central nervous system because serum ceruloplasmin does not cross the blood brain barrier. The brain areas that undergo the most severe iron overload in aceruloplasminaemia are those that normally contain the highest amounts of this metal: the basal ganglia, the red nuclei, and the deep cerebellar nuclei. ${ }^{12}$ Surprisingly, no brain iron overload occurs in a mouse model for aceruloplasminaemia that only shows accumulation of this metal in reticuloendothelial cells and hepatocytes, pointing out important species differences in iron handling. ${ }^{6}$ The same mouse model demonstrates no copper metabolism abnormality, $^{10}$ this time in accordance with the human disease, despite very low serum copper. This clearly indicates that copper transport is just an incidental function of ceruloplasmin, related to its abundance in serum and its need of copper as prosthetic group.

Here we describe a case of aceruloplasminaemia that was diagnosed early in the symptomatic course of the disease. This case very well illustrates how iron overload can be tolerated by brain cells for a long time with no pathological consequences. After the iron storage capacity of neural and glial cells is exceeded, neural dysfunction and neurodegeneration rapidly occur.

\section{METHODS \\ Patient}

The patient was a 59 year old lady of Belgian origin who came to the Erasme Hospital in Brussels because of focal dystonia developed over the previous two years.

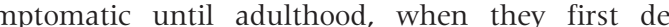
diabetes mellitus. A progressive neurological disease begins a few years later, typically with focal dystonia, followed by dysarthria, ataxia, bradykinesia, rigidity, and dementia. Evidence of hepatic iron overload and peripheral retinal degeneration are present, but usually have little clinical impact. Laboratory studies reveal microcytic anaemia, hyperferritinemia, absent serum ceruloplasmin, and very low copper. ${ }^{1}$

Ceruloplasmin is a serum multicopper ferroxidase. ${ }^{3}$ Though it carries most serum copper; its major role is in iron rather than in copper metabolism. It is thought to promote iron efflux out of several cell types, probably by generating ferric iron to be inserted into apotransferrin..$^{3-6}$ Lack of ceruloplasmin causes iron accumulation in neurones and astrocytes of the brain, in the retinas, in parenchymal and reticuloendothelial cells of the liver, in reticuloendothelial cells of the spleen, and in pancreatic cells. ${ }^{1-4}$ Anaemia may occur because iron is not efficiently delivered to red blood cell precursors. Ceruloplasmin gene expression specific to astrocytes has been demonstrated in the mammalian brain and in some species, including human beings, ${ }^{7}$ may be critical for iron metabolism and neuronal survival in the retina and basal ganglia. ${ }^{3}$ Ceruloplasmin in astrocytes is in part directly anchored to

\section{FDG-PET scan}

Brain positron emission tomography (PET) was performed on a CTI-Siemens HR+ tomograph using ${ }^{18} \mathrm{~F}$-fluorodeoxyglucose (FDG) to study distribution of cerebral glucose metabolism. Voxel based analysis comparing patient's cerebral glucose metabolism with that of 29 right handed healthy controls (16 women and 13 men, mean age, 32) was performed using Statistical Parametric Mapping (SPM99) (Wellcome Department of Cognitive Neurology). Data from each subject were normalised to a standard stereotactic space and then smoothed with a $12 \mathrm{~mm}$ full width half maximum isotropic kernel. The analysis identified brain regions where glucose metabolism was significantly altered in the patient scan

Abbreviations: $\mathrm{CT}$, computed tomography; DFO, desferioxamine; glycosylphosphatidylinositol; MRI, magnetic resonance imaging; PET,
positron emission tomography; PANK2, an isoform of pantothenate kinase; PKAN, pantothenate kinase associated neurodegeneration FDG-PET, fluorodeoxyglucose positron emission tomography; GPI, 
compared with the control group. Results were considered significant at $\mathrm{p}<0.05$ corrected for multiple comparisons over the entire brain volume. Considering an a priori hypothesis of basal ganglia dysfunction, in these structures we also considered results significant at $\mathrm{p}<0.05$ after a conservative (radius $50 \mathrm{~mm}$ ) small spherical volume correction.

\section{Molecular genetic analysis}

Genomic DNA was extracted from venous blood using the Puregene kit (Gentra) according to the manufacturer's instructions. The ceruloplasmin gene's 19 exons $^{11}$ were amplified and directly sequenced as described previously. ${ }^{2}$

\section{RESULTS}

\section{Case report}

The patient had been diagnosed with seronegative rheumatoid arthritis at the age of 42 and with diabetes mellitus at the age of 55. She had had mild hypochromic anaemia throughout her life. The first neurological symptom was an increased blinking frequency at age 57. Tonic closure of the eyelids, involuntary mouth opening, jaw clenching, and lips and tongue protrusion then appeared and progressed. About one and a half years later, she started to feel dizzy and unstable, but she never fell. She was examined at age 59, two years after the onset of the symptoms. The patient had not taken dopamine receptor blocking agents in the year prior to the onset of dystonia. General physical examination was unremarkable; in particular no sign of active rheumatoid arthritis was detected. Neurological examination revealed a bilateral blepharospasm and oromandibular dystonia. Gait and balance were considered normal despite the subjective complaints of the patient. She appeared somewhat clumsy, but she had no dysmetria or intention tremor. The rest of the neurological exam was unremarkable; in particular there was no eye movement abnormality and no motor or sensory deficit. The patient had no obvious cognitive deficit and her MMSE score was 30/30. There was no corneal KayserFleischer ring. A mild peripheral pigmentary retinal degeneration was detected at the fundoscopic examination.

Brain computed tomography (CT) showed a mildly increased density of the basal ganglia (figure le). T2 weighted magnetic resonance imaging (MRI) of the brain showed a symmetrical severe signal loss in the caudate, putamen, red nucleus, dentate nucleus, and posterior thalamus; a less severely decreased signal in the pallidum; and a low signal ribbon all along the cerebral cortex (figures la-d). A similar loss of T2 signal was found in the liver at abdominal MRI (figure lf).

Routine blood tests confirmed a poorly controlled diabetes mellitus. Haemoglobin, white blood cell counts and red blood cell counts were normal with a normal red blood cell mean corpuscular volume. Erythrocyte sedimentation rate and C reactive protein were normal, rheumatoid arthritis (RA) serology was negative. The patient had marked hyperferritinaemia $(1900 \mathrm{ng} / \mathrm{ml})$ with serum iron in the low normal range and low transferrin saturation. Serum ceruloplasmin and copper were below the laboratory detection limits.

\section{PET analysis}

FDG-PET showed a relative decrease of metabolism in the head of left and right caudate nucleus (figure 2). After spherical volume correction, voxel level $p$ values were 0.006 and 0.02 in the left and right caudate nucleus respectively; cluster level p values were 0.02 on both sides. Only one cortical cluster of voxels approached the level of significance as defined here. This cluster was located in the posterior part of the anterior cingulate cortices $(\mathrm{p}<0.001$ at uncorrected voxel level; $p=0.14$ and 0.01 , respectively at voxel and cluster levels after correction for multiple comparisons over the entire brain volume).

\section{Molecular genetic studies}

Two novel ceruloplasmin gene mutations were found in our patient: (a) Nt 146(+1) G to A at 3' splice site of exon 1, and (b) Nt $2602 \mathrm{l} \mathrm{bp} \mathrm{deletion} \mathrm{in} \mathrm{exon} \mathrm{15.} \mathrm{Both} \mathrm{mutations} \mathrm{result} \mathrm{in}$ premature stop codons, which usually lead to rapid mRNA degradation and no protein synthesis. Even if some ceruloplasmin were made by our patient, both mutations are predicted to truncate the protein so that it will lack its carboxy terminal portion containing critical ligands for the trinuclear copper cluster essential for ferroxidase activity. ${ }^{12}$

\section{DISCUSSION}

Our patient clearly illustrates the early neurological manifestations of aceruloplasminaemia. Clinical and PET findings show how dysfunction is initially limited to the caudate nucleus, despite an already severe and widespread iron
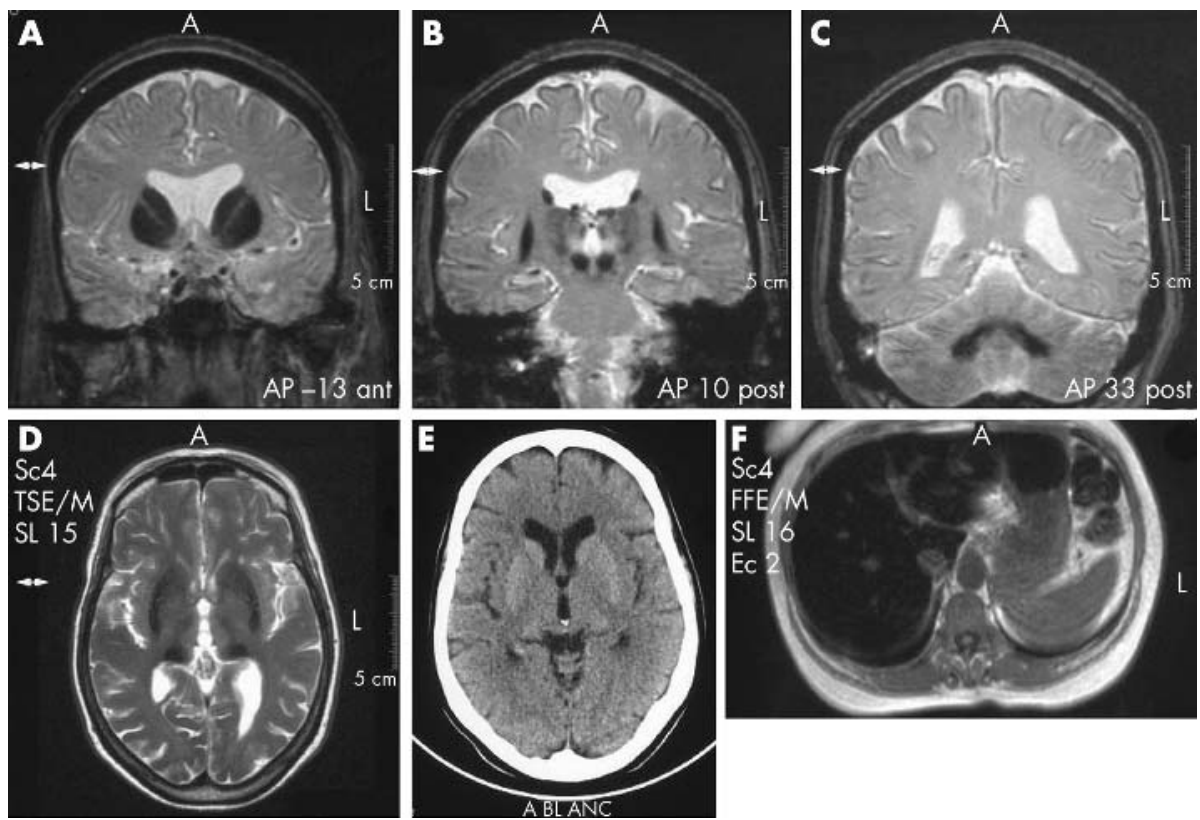

Figure 1 Gradient echo $(A, B)$ and T2 weighted $(C, D)$ brain MRI images showing a symmetrical very low signal intensity in the dentate nuclei, red nuclei, putamen, caudate nuclei, posterior thalami, and a low signal ribbon all along the cerebral cortex. Notice the normal T2 signal intensity in the pallidum. A computed tomogram of the brain (E) shows a mild, homogeneous hyperdensity of the caudate, putamen, and posterior thalamus bilaterally. Abdominal MRI reveals a marked hepatic iron accumulation as loss of signal in T2 weighted images (F). 

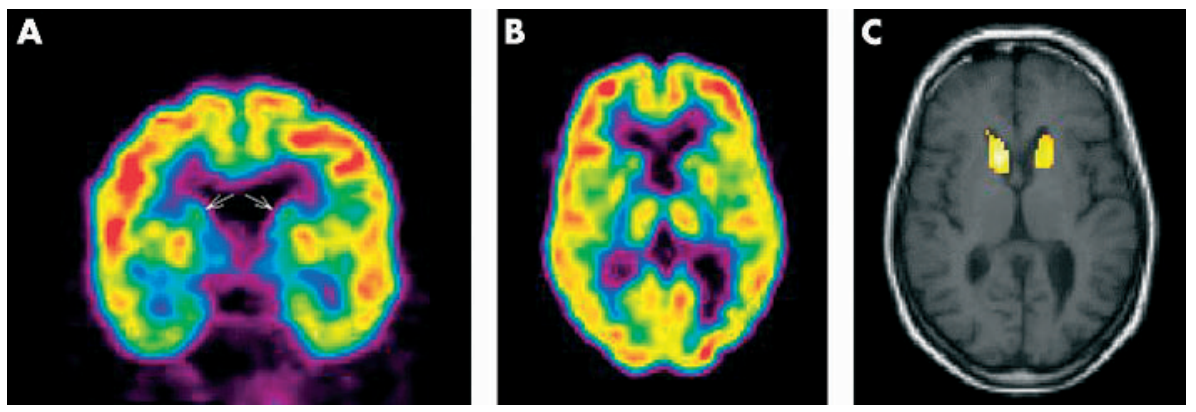

Figure 2 Native reconstructed PET slices in coronal (A) and transaxial (B) planes through the caudate nuclei (arrows). (C) Results of the SPM99 analysis showing a decreased metabolism in the heads of caudate nuclei. The dysfunctional brain areas are presented on a coregistrated T1 magnetic resonance image of the patient.

accumulation in various regions of the brain. It appears that the brain can store very large amounts of this metal before its capacity is exceeded, resulting in toxicity and rapid neurodegeneration. In advanced cases of aceruloplasminaemia, iron overload and extensive neuronal loss are found particularly in the basal ganglia and deep cerebellar nuclei, therefore the regional distribution of brain iron overload explains the extrapyramidal and ataxic features of the disease. ${ }^{13}$ Even though pathological studies showed little iron accumulation in the cortex, our patient's MRI scan shows that it occurs as well, probably contributing to the late dementia. The pathology of aceruloplasminaemia bears a resemblance with two other brain iron overload diseases: neuroferritinopathy ${ }^{14}$ and pantothenate kinase associated neurodegeneration (PKAN). ${ }^{15}$ However, aceruloplasminaemia and neuroferritinopathy are adult onset diseases, while PKAN differs by having its onset in childhood or, in atypical cases, during adolescence. We hypothesise that these differences in age of onset relate to different mechanisms of iron overload. Iron accumulation in PKAN is thought to be secondary to cysteine accumulation resulting from the underlying genetic enzymatic defect in the pantothenate kinase isoform most represented in the basal ganglia (PANK2). ${ }^{15}$ PANK2 normally generates phosphopantothenate that then condenses with cysteine in the coenzyme A synthesis pathway. Cysteine has a tendency to bind iron and the resulting complex has redox activity that can generate toxic free radicals. Conversely, in the adult onset diseases, iron first accumulates in a non-toxic form within a ferritin shield that protects it from the cellular environment, preventing free radical generating redox reactions. In aceruloplasminaemia, ferritin is eventually filled to capacity because of the reduced ability of the cell to extrude iron. In neuroferritinopathy, abnormal ferritin-iron complexes form because of a genetic abnormality of ferritin L chains, triggering iron deposition through a still unknown mechanism. ${ }^{14}$ Our case illustrates very well the remarkable iron storage capacity of the brain, particularly but not exclusively of the basal ganglia. It is only when ferritin storage capacity is exceeded that the now unprotected iron can be engaged in redox reactions, generate free radicals and cause pathology. In this regard, there is evidence of lipid peroxidation ${ }^{16}$ and oxidative damage to respiratory chain complexes $^{17}$ in aceruloplasminaemia brains. Probably just a small excess of iron beyond ferritin capacity is sufficient to cause rapid neurodegeneration, as suggested by the relentlessly progressive and relatively rapid course of symptomatic aceruloplasminaemia and neuroferritinopathy.

While in more advanced cases of aceruloplasminaemia PET showed a marked decrease in glucose and oxygen consumption in the entire brain, ${ }^{18}$ in our patient the only detectable metabolic abnormality by FDG-PET was a relative hypometabolism of the caudate nuclei. It is tempting to correlate this finding with the clinical picture of blepharospasm and oromandibular dystonia. Previous FDG-PET studies in patients with focal dystonia, in particular blepharospasm, were mostly carried out in idiopathic cases. Results have been conflicting, possibly because of methodological and patient selection issues, however several studies reported hypometabolism in the striatum and in cortical motor areas controlling the dystonic muscles as baseline, movement unrelated abnormalities. ${ }^{19}$ The findings in our patients suggest that primary caudate nucleus involvement by a neurodegenerative process leading to hypometabolism in this structure result in focal cranial dystonia. Intriguingly, the posterior part of the anterior cingulate cortex may be affected as well, probably as a secondary phenomenon to caudate dysfunction.

Our patient also presented with a history of seronegative polyarthritis. We found no case report of arthritis associated with aceruloplasminaemia, but iron overload due to hemochromatosis can cause polyarthropathy mimicking rheumatoid arthritis. Our patient developed a polyarthropathy of the wrist, foot, knee, and fingers in her forties. Hand and foot radiographies showed mild bilateral osteoarthritis of the first metatarsophalangeal joints. No specific aetiology could be determined for the patient's arthropathy, despite repeated testing. We suggest the possibility that iron overload due to aceruloplasminaemia may also affect the joints, causing symptoms similar to the hemochromatosis-associated arthropathy.

Treatment was attempted with desferioxamine (DFO), as suggested by Myajima et al. ${ }^{20}$ The possibility of early intervention, when most brain regions have yet to accumulate toxic amounts of iron, gave us hope for an improvement in the long term outcome of this patient. After six months of DFO, at a dosage of $2400 \mathrm{mg}$, four times a day, 5 days a week by subcutaneous infusion pump, there was no further progression of neurological symptoms, ferritinaemia fell to about $700 \mathrm{ng} / \mathrm{ml}$, but diabetes control was not improved. The patient did not experience any systemic side effect of DFO and was not rendered anaemic by the treatment. However, continuation of DFO treatment is becoming difficult because of complications at the injection site.

A final consideration concerns the finding that our patient is a compound heterozygote for two different loss of function ceruloplasmin mutations. Given the supposed rarity of the disease, this is surprising. Most cases of rare recessive disorders are thought to be the result of inbreeding, whether or not there is known consanguinity between the patient's parents, which implies homozygosity for the causative mutation. This has indeed been the case for aceruloplasminaemia, particularly in Japan, which is the country where most cases so far reported originate. ${ }^{21}$ Compound heterozygosity in our patient, who is to our knowledge the first reported Belgian case, suggests that aceruloplasminaemia may be less rare than expected in European populations and may therefore be underdiagnosed. This finding is particularly interesting in the light of the report of cerebellar ataxia segregating with heterozygous ceruloplasmin mutations in four Japanese families. ${ }^{22}$ The possibility that such mutations 
may be more common in Europe than previously thought suggests that it may be worthwhile screening European patients with unclassified familial cerebellar ataxia for hypoceruloplasminaemia.

\section{Authors' affiliations}

I Haemers, M Pandolfo, Department of Neurology, Hôpital Erasme, Université Libre de Bruxelles, Brussels, Belgium

S Goldman, PET/Biomedical Cyclotron Unit, Hôpital Erasme, Université Libre de Bruxelles, Brussels, Belgium

S Kono, J D Gitlin, Department of Pediatrics, Washington University School of Medicine, St Louis, Missouri

Competing interests: none declared

Correspondence to: $\operatorname{Dr} M$ Pandolfo, Service de Neurologie, Hôpital Erasme, Route de Lennik 808, 1070 Bruxelles, Belgium; massimo.pandolfo@ulb.ac.be

Received 24 April 2003

In revised form 26 June 2003

Accepted 11 July 2003

\section{REFERENCES}

1 Nittis T, Gitlin JD. The copper-iron connection: hereditary aceruloplasminemia. Semin Hematol 2002;39:282-9.

2 Yoshida K, Furihata K, Takeda S, et al. A mutation in the ceruloplasmin gene is associated with systemic hemosiderosis in humans. Nat Genet 1995;9:267-72.

3 Hellman NE, Gitlin JD. Ceruloplasmin metabolism and function. Annu Rev Nutr 2002;22:439-58.

4 Qian ZM, Ke Y. Rethinking the role of ceruloplasmin in brain iron metabolism. Brain Res Brain Res Rev 2001;35:287-294.

5 Harris ZL, Takahashi Y, Miyajima H, et al. Aceruloplasminemia: molecular characterization of this disorder of iron metabolism. Proc Natl Acad Sci U S A 1995; $92: 2539-43$.
6 Harris ZL, Durley AP, Man TK, et al. Targeted gene disruption reveals an essential role for ceruloplasmin in cellular iron efflux. Proc Natl Acad Sci U S A 1999;96:10812-7.

7 Klomp LW, Gitlin JD. Expression of the ceruloplasmin gene in the human retina and brain: implications for a pathogenic model in aceruloplasminemia. Hum Mol Genet 1996;5:1989-96.

8 Patel BN, David S. A novel glycosylphosphatidylinositol-anchored form of ceruloplasmin is expressed by mammalian astrocytes. J Biol Chem 1997;272:20185-90.

9 Patel BN, Dunn RJ, David S. Alternative RNA splicing generates a glycosylphosphatidylinositol-anchored form of ceruloplasmin in mammalian brain. J Biol Chem 2000;275:4305-10.

10 Meyer LA, Durley AP, Prohaska JR, et al. Copper transport and metabolism are normal in aceruloplasminemic mice. J Biol Chem 2001;276:36857-61.

11 Daimon M, Yamatani K, Igarashi M, et al. Fine structure of the human ceruloplasmin gene. Biochem Biophys Res Commun 1995;208:1028-35

12 Hellman NE, Kono S, Mancini GM, et al. Mechanisms of copper incorporation into human ceruloplasmin. J Biol Chem 2002;277:46632-8.

13 Kaneko K, Yoshida K, Arima K, et al. Astrocytic deformity and globular structures are characteristic of the brains of patients with aceruloplasminemia. J Neuropathol Exp Neurol 2002;61:1069-1977.

14 Curtis AR, Fey C, Morris CM, et al. Mutation in the gene encoding ferritin light polypeptide causes dominant adult-onset basal ganglia disease. Nat Genet $2001 ; 28: 350-4$.

15 Zhou B, Westaway SK, Levinson B, et al. A novel pantothenate kinase gene (PANK2) is defective in Hallervorden-Spatz syndrome. Nat Genet $2001 ; 28: 345-9$.

16 Yoshida K, Kaneko K, Miyajima H, et al. Increased lipid peroxidation in the brains of aceruloplasminemia patients. I Neurol Sci 2000;175:91-6.

17 Kono S, Miyajima H, Takahashi Y, et al. Defective electron transfer in complexes I and IV in patients with aceruloplasminemia. J Neurol Sci 2000:182:57-60.

18 Miyajima H, Takahashi Y, Kono S. Glucose and oxygen hypometabolism in aceruloplasminemia brains. Intern Med 2002;41:186-90.

19 Hutchinson M, Nakamura T, Moeller JR, et al. The metabolic topography of essential blepharospasm: a focal dystonia with general implications. Neurology 2000;55:673-7.

20 Miyajima H, Takahashi Y, Kamata T, et al. Use of desferrioxamine in the treatment of aceruloplasminemia. Ann Neurol 1997;41:404-7.

21 Miyajima H, Kono S, Takahashi Y, et al. Estimation of the gene frequency of aceruloplasminemia in Japan. Neurology 1999:53:617-9.

22 Miyajima H, Kono S, Takahashi $\mathrm{Y}$, et al. Cerebellar ataxia associated with heteroallelic ceruloplasmin gene mutation. Neurology 2001;57:2205-10. 